Communications in Physics, Vol. 22, No. 3 (2012), pp. 275-281

\title{
A FEASIBILITY STUDY IN APPLICATION OF A GAMMA SCATTERING TECHNIQUE FOR INSPECTING DENSITY VARIATION BY MONTE CARLO METHOD
}

\author{
LE HONG KHIEM, TRAN DINH TRONG \\ Center for Nuclear Physics, Institute Of Physics, VAST \\ NGUYEN VAN THAO \\ Center of Technology Development Assistance and Service, VAST
}

\begin{abstract}
Back-scattering gamma-rays have been extensively used for years as a nondestructive tool for inspecting the materials in different fields of the economy. The intensities of Compton scattering gamma-rays from the scattering medium strongly depend on its electron density and therefore in its mass density. This feature is very useful for using these rays as a viable tool for inspecting material. This work aims to investigate the feasibility of application of gamma scattering technique for inspecting density variation in some construction objects by Monte-Carlo simulation method. The gamma-ray sources of different energies and strengths have been used to extract the information of density variation for interior of sample by recording the backscattering gamma-rays with a gamma-ray detector. The results of our simulations confirm that the resolution for density variation in the inspected objects is quite good. The results should also prove useful in the optimum design of the nondestructive density gauges.
\end{abstract}

\section{INTRODUCTION}

Back-scattering gamma rays have been extensively used for years as a nondestructive tool for inspecting the materials in industrial non-destructive testing instruments and techniques [1-3]. The intensities of Compton scattering gamma-rays from the scattering medium strongly depend on its electron density and therefore on its mass density. This feature is very useful for using back-scattering gamma-rays as a viable tool for inspecting material, especially for ascertaining quantitative information of density of an inspecting object. For example, in highway construction, two aspects are very important for the quality of the road and have to be controlled, namely asphalt compaction and density. In the world, many nuclear instruments for measuring in-situ density of materials are available from various manufacturers and nowadays they are widely used for density testing. The asphalt industry, for example, has been forced to use nuclear density gauges for measuring the quality of the asphalt that has been placed, especially for inspecting the compaction process and density of the asphalt layer. Densification of asphalt during the construction of highways is one of the key aspects of the road building process.

The main advantage of nuclear methods is that they are rapid, non-destructive and continual measurement in comparison with the conventional density tests. Moreover, they offer greater degree of freedom from human error and require less judgment on the part of the operation. On the other hand, nuclear methods have several disadvantages. For 
example, the determination of density using nuclear methods is indirect. As a result, calibration relations have to be developed. These calibration relations may vary with different soils depending on their chemical composition, heterogeneity and surface texture. Therefore, calibration curves developed by the manufacturers for a specific type of soil might not be valid under conditions different from those that were used in the original development. In addition, the equipment utilizes radioactive materials which may be hazardous to the health of the users unless proper precautions are taken. Despite these disadvantages, the gamma-ray surface backscattering technique has been widely applied for measuring the density of pavement and the apparatus has been named as a nuclear density gauge.

In Vietnam, the testing techniques for asphalt density are based on one founded hundreds of years ago. It involves extracting a core sample and using Archimedes principle of water displacement to measure pavement density. This water displacement test will be the test of record for the project and will be performed at random intervals during the construction process. This test is destructive, very time consuming and is becoming obsolete. Some imported nuclear instruments for density testing exit. However, they are not widely used due to their very high costs. We are now planning to build an apparatus using back-scattering gamma-ray technique for density testing in Vietnam. As a first stage of the plan, it is necessary to study feasibility of the method by simulation. We have made a comprehensive study of the dependence of the scattered flux on the primary gammaray energy, material atomic number and geometrical configuration, by using Monte-Carlo method. We shall describe and discuss the computational method as well as the results obtained for different types of geometric configuration. From these results, we are able to choose the best conditions for designing the apparatus.

\section{SIMULATION METHOD}

The schematic geometry configuration used in simulation is shown in Fig. 1. The employed geometry involves a radioactive source with gamma-ray energy usually less than $1300 \mathrm{keV}$ and detector system placed on the surface of the layer to be tested. The source separates from the detector by lead shielding. The shielding prevents the gamma-rays coming directly from the source to the detector. In these circumstances, the gamma-rays from the source pass down into the layer under test, suffer interactions with matter and are back-scattered onto a detector a distance d from the source. In addition, because of further interactions, the beam is continuously attenuated in both the forward and the backward passages through the layer. In general, the relevant absorption coefficient is made up of the absorption due to the photoelectric effect, the scattering due to the Compton Effect and the absorption due to pair production. However, since energy of primary gamma-ray is less than $1300 \mathrm{keV}$, the contribution of pair production can be neglected. Thus only photoelectric absorption and Compton scattering were accounted for. The intensity of the detected gamma-rays which is measured depends on the gamma-ray scattering and absorbing properties of the material beneath the probe. A calibration curve relating the intensity of the detected radiations to density is usually constructed by using samples of the same chemical composition but with different densities. 


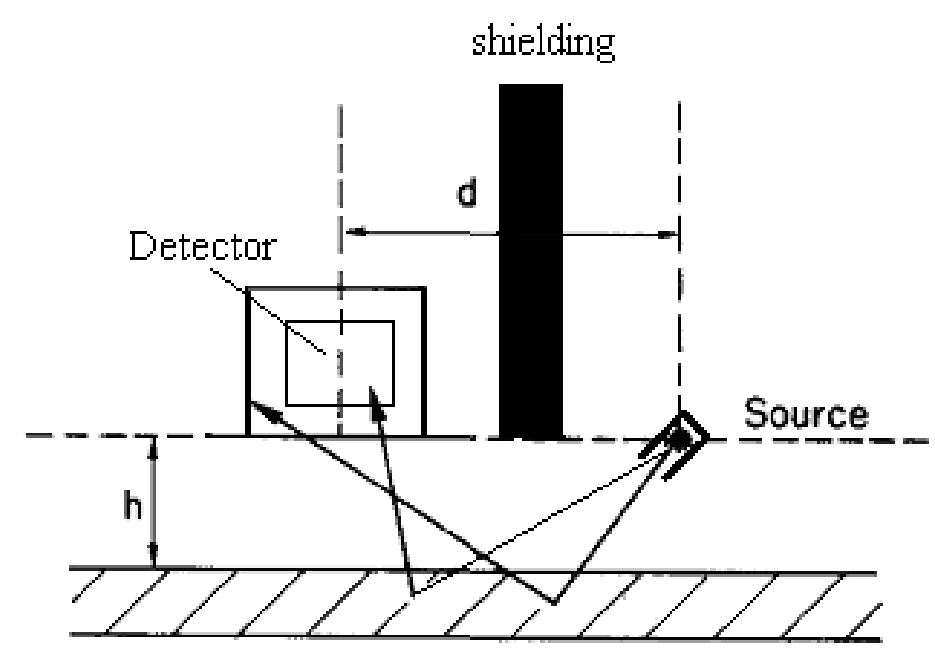

Fig. 1. The schematic geometric configuration used in the simulation

The Monte-Carlo method used in our calculation is similar to the one previously described in [4-6]. Our simulation is limited to the primary gamma-ray energies in the range from 0.2 to $1.3 \mathrm{MeV}$. The program simulates the interaction of each gamma-ray entering the material. The point of interaction of the gamma-ray is determined randomly according to the total gamma-ray interaction cross section. The type of interaction is also determined randomly using the relative values of the cross sections involved. If the gamma-ray interacts with the material atom via photoelectric effect then it is assumed to be absorbed and its history will be terminated.

In Compton scattering a gamma ray of energy $E$ is scattered by one of the free or loosely bound electrons of the material. Following the interaction between gamma-ray and electron, the gamma-ray is then scattered at an angle $\theta$ with respect to its initial direction with an energy $E^{\prime}$ and the electron recoils. The gamma ray is scattered off in a new direction with a lower energy. Values for $E^{\prime}$ can be obtained by sampling the KleinNishina formula. By knowing the $E^{\prime}$, the scattering angle $\theta$ of the scattered gamma ray can be easily determined [7].

The differential cross sections and total cross-sections for collisions between the incident gamma and electron can be calculated by the Klein-Nishina formula.

The probability for Compton scattering varies approximately as the atomic number of the scattering medium and decreases as the energy of the gamma ray increases. A Compton recoil electron and the bremsstrahlung are the secondary radiations in a Compton scattering event. The formula for $E^{\prime}$ is an inverse function of the Klein-Nishina differential cross-section and is given due to Carlson by:

$$
E^{\prime}=\frac{E}{1+S q+(2 E-S) q^{3}}
$$


where, $S=E /(1+0.5625 E)$ and $q$ is a random number between 0 and 1 . The gamma-ray is allowed to diffuse in the material until they escaped, or its energy is less than $20 \mathrm{keV}$. A detailed algorithm for simulation can be found in [8].

A computer program named NUCLGAUGE was developed using Monte-Carlo method for simulation of backscattered gamma-ray intensities measured under the geometrical configuration mentioned above. The program has been written in $\mathrm{C}++$. By using this program, we are able to investigate the dependence of the back-scattered gamma-ray intensities on various parameters needed to be optimized.

\section{RESULTS OF SIMULATION}

Below we will describe the results of various simulations performed for a design of a density thickness using NUCLGAUGE program. The gamma-ray source is assumed to be ${ }^{137} \mathrm{Cs}$ and $\mathrm{NaI}$ detector will be used for registration of the backscattered gamma-rays

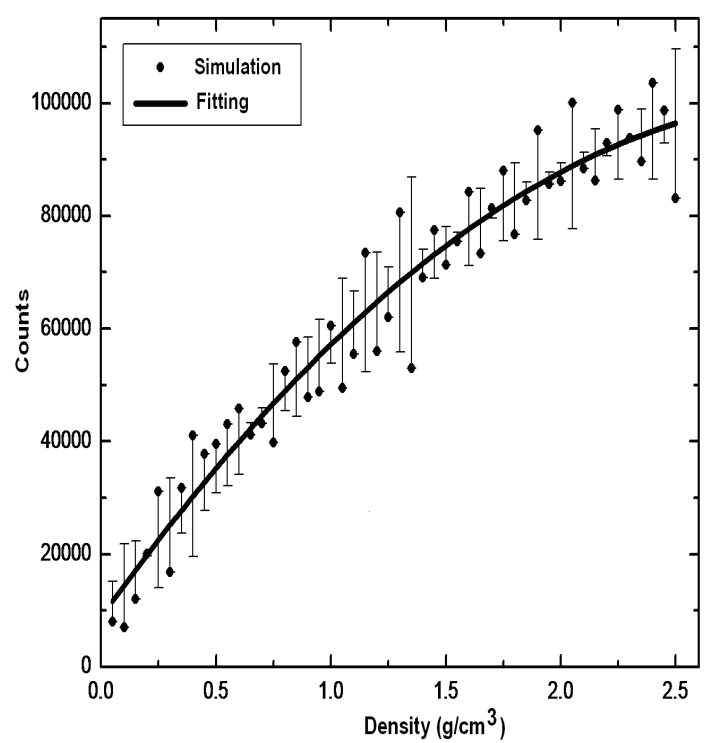

Fig. 2. Dependence of the backscattered gamma-ray intensity on the density of material.

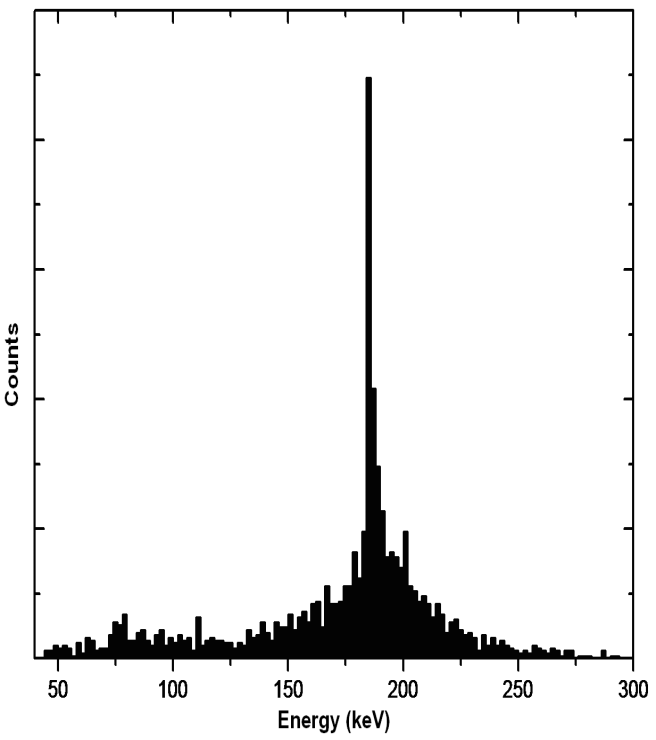

Fig. 3. Energy distribution of the backscattered gamma-rays registered by the detector.

\section{1) Dependence of the backscattered gamma-ray intensities on the density of material}

A typical result of the dependence of backscattered gamma-ray intensity on the material densities is presented in Fig. 2. In this simulation, we assumed that the intensity of the ${ }^{137} \mathrm{Cs}$ gamma source is $1 \mathrm{mCi}$. The diameter of the source collimator is $2 \mathrm{~cm}$ and the inclined angle to the vertical direction is $45^{\circ}$. The diameter of NaI crystal is $6 \mathrm{~cm}$ with $6 \%$ of efficiency. Separation between detector and source is $5 \mathrm{~cm}$ and the distance from detector to the surface of material layer is $5 \mathrm{~cm}$. The investigated material is soil with an effective charge number of 10 and effective mass number of 21 . It is clearly seen 
from Fig. 2 that the backscattered gamma-ray intensity linearly depends on the material density up to $2.0 \mathrm{~g} / \mathrm{cm}^{3}$. Therefore, it is possible to measure the variation of material density by measuring the backscattered gamma-rays.

\section{2) Simulation of energy of the backscattered gamma-rays registered by the detector}

The energy distribution of the backscattered gamma-rays registered by the detector is presented in Fig. 3. Most of the backscattered gamma-rays have an energy around 175 $\mathrm{keV}$ to $200 \mathrm{keV}$. This result is in agreement with the predicted one calculated by KleinNishina formula. From the figure, we can easily choose an optimal energy window of the detector in order to eliminate the background gamma-rays coming from other sources.

\section{3) An optimal radius of the source collimator for a fixed size of the detector}

We assume that the size of the detector is fixed with the diameter of its crystal is $5 \mathrm{~cm}$ and $5 \mathrm{~cm}$ height. We also assume that the source is in the form of a disk whose radius is $80 \%$ of the inner-radius of the collimator. Suppose the thickness of the lead shield between the detector and the source is thick enough for completely shielding $661 \mathrm{keV}$ of the ${ }^{137} \mathrm{Cs}$ source. We hope to determine the optimal radius of the collimator with which the detector efficiency becomes maximal. When calculations are performed for collimators of radius $1 \mathrm{~cm}, 2 \mathrm{~cm}, \ldots, 20 \mathrm{~cm}$, each with 50000 gamma-rays, we obtained the results shown in Fig. 4. Note that in the range of the collimator radius from 1 to $20 \mathrm{~cm}$, the wider the collimater is, the higher efficiency can be obtained. However for practical designing, the size of radius must be kept in such a way that the efficiency and the weight of apparatus must be optimal.

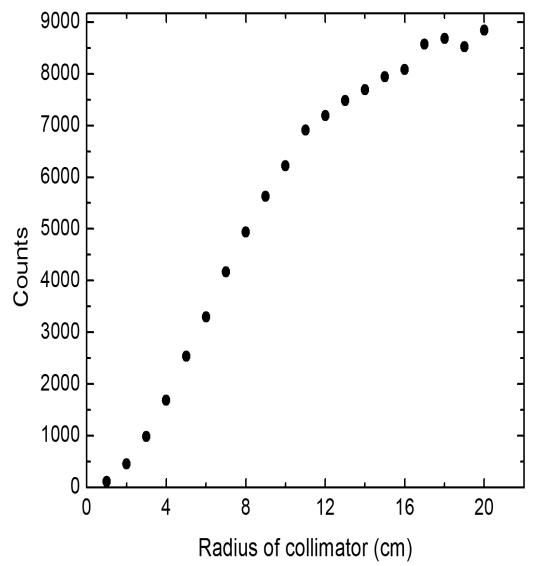

Fig. 4. Detected back-scattered gamma-ray intensity as a function of the radius of source collimator.

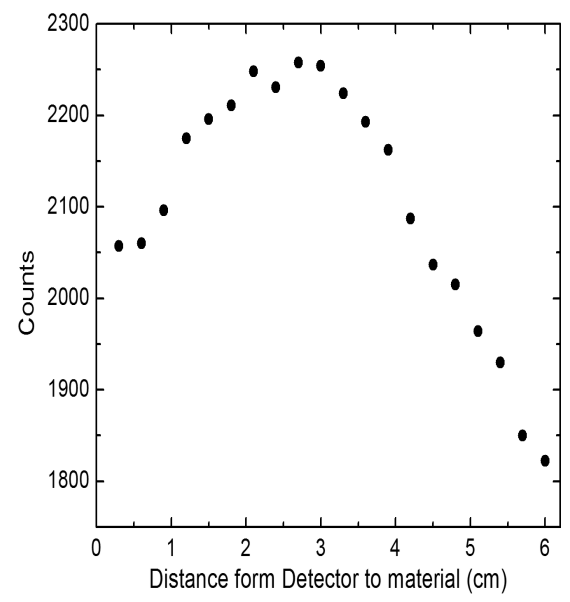

Fig. 5. Detected back-scattered gamma-ray intensity as a function of the distance between detector and material layer. 


\section{4) An optimal distance from the detector to the material}

In order to optimize the distance from the detector to the material, we have to simulate using various distances from the detector to the object material for finding how the spectrum changes as the distance varies. Again, we fixed the size of the detector to be $5 \mathrm{~cm}$ in diameter and $5 \mathrm{~cm}$ height. The optimal diameter of the collimator is assumed to be $0.7 \mathrm{~cm}$. A set of simulations has been carried out for the 20 different cases with distances ranging from $1 \mathrm{~mm}$ to $20 \mathrm{~mm}$. The results are shown in Fig. 5 .

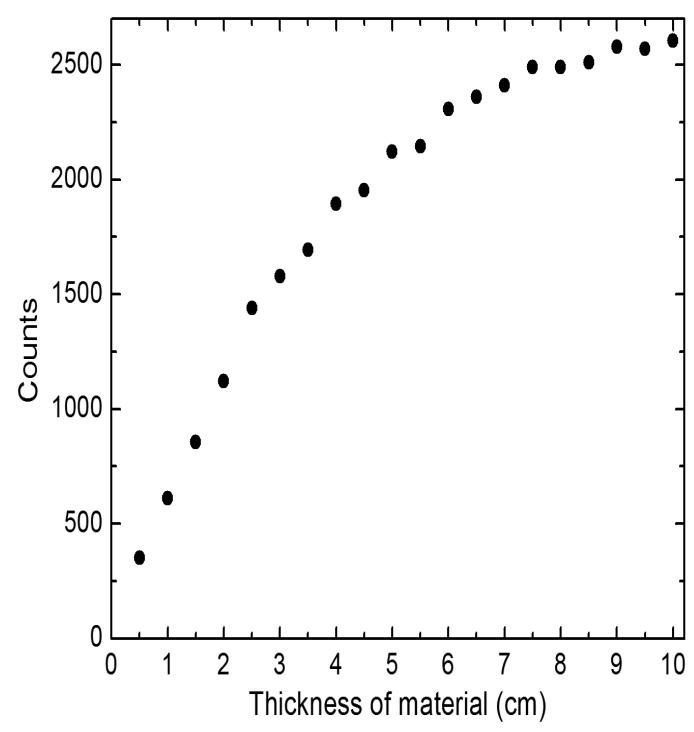

Fig. 6. Detected back-scattered gamma-ray intensity as a function of material thickness

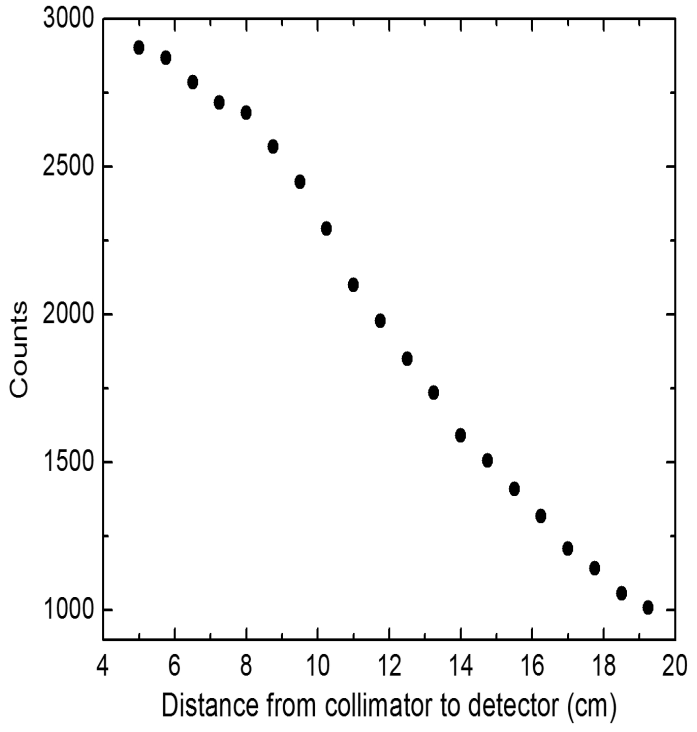

Fig. 7. Detected back-scattered gamma-ray intensity as a function of source collimatordetector distance.

\section{5) The thickness versus the radiation count}

In order to see how the number of detected gamma changes as the thickness of the material layer increases, we performed a series of simulations for a fixed material density and geometry. The thickness varies from $1 \mathrm{~cm}$ to $10 \mathrm{~cm}$ with the step of $0.5 \mathrm{~cm}$. The results are shown in Fig. 6. One can see that the total number of counts measured by the detector increases as the thickness increases in the range from 0 to $6 \mathrm{~cm}$. Over this range, we are not able to use this technique. Therefore for measuring density by this technique, we have to assume that the material under investigation must have the same density over the investigated volume.

\section{6) The detected back-scattered gamma-ray intensity as a function of detector-} source collimator distance

This dependence is shown in Fig. 7. It is clearly seen from the figure that the back-scattered gamma-ray intensity decreases as the detector-source distance increases. However, if this distance is to short then the background due to primary gamma-ray from 
the source will increase. Therefore, in designing we have to optimize these two parameters in order to maximize the effiency of the apparatus while the background from the source is not so high.

\section{CONCLUSIONS}

By using the Monte-Carlo method, we have studied the dependence of the backscattered gamma-ray intensity on various parameters of the proposed apparatus for density measurement based on Compton scattering. The NUCLGAUGE computer program has been developed by our group for the optimazation of the design. Application of thisprogram to the problem of gamma-ray backscattering allows us to obtain a realistic dependence of the backscattered flux on the primary gamma-ray energy and material atomic number for different geometrical configurations. Furthermore, the results of the energy dependence of the backscattered flux also allow us to choose the optimal gamma-ray energy for a particular material under investigation. From our simulation results, the application of the gamma-ray backscattering method for in situ measurement of the density profile is feasible with some limitations. The main errors arise from density gradients and from differences in the relation between the back-scattered intensity and density for different materials. Reduction of these errors requires an understanding of the depth-sampling characteristic of the apparatus. Our future plan is to produce a prototype density apparatus based on our study under financial support of some foundation.

\section{ACKNOWLEDGEMENT}

The authors would like to thank the Center of Technology Development Assistance and Service, Vietnam Academy of Science and Technology for partially financial supporting our work.

\section{REFERENCES}

[1] K. Preiss and A. Haccoun, Nuclear Engineering and Design 30 (1974) 123.

[2] Esam M. A. Hussein, Tim M. Whynot, Nuclear Instruments and Methods in Physics Research $\mathbf{A 2 8 3}$ (1989) 100.

[3] B. S. Moon, Y. K. Kim, J. Y. Kim, J. T. Kim, C. E. Chung, S. B. Hong, Journal of the Korean Nuclear Society 32(5) (2000) 457.

[4] Le Hong Khiem, Nguyen Van Do, Pham Duc Khue, International J. Radioanal. Nucl. Chem., Lett. 200(4) (1995) 299.

[5] Le Hong Khiem, Data Analysis in Radiation Measurement, Vietnam National University Publishing House, Hanoi (2008) (in Vietnamese).

[6] . Le Hong Khiem, Nguyen Tuan Khai, Proceedings of 15th World Conference on Non - Destructive Testing, 15 - 21 October 2000 in Rome (http:// www.ndt.net/article/ wcndt00/ index.htm).

[7] . K. S. Krane, Introductory Nuclear Physics, John Wiley \& Sons Inc. (1988).

[8] . Le Hong Khiem, Gamma-Ray Spectrometry, Vietnam National University Publishing House, Hanoi (2010) (in Vietnamese).

Received 22 April 2011. 